\title{
Contamination of specimen container surfaces during sputum collection
}

\author{
BW ALLEN, JH DARRELL* \\ From Medical Research Council Unit for Laboratory Studies of Tuberculosis, and the *Department of \\ Bacteriology, Royal Postgraduate Medical School, Du Cane Road, London W12 OHS
}

SUMMARY Sputum specimens from culture-positive tuberculosis patients were examined for the presence of Myobacterium tuberculosis on container surfaces. Although specimens were in transit for several days, $M$ tuberculosis was isolated from $18(6.5 \%)$ of 279 containers examined. Sputum specimens from local patients were examined for evidence of upper respiratory bacterial flora on the outside of containers as an indicator of contamination with sputum. Of 300 containers examined, $41(14 \%)$ were contaminated. A technique for disinfecting specimen containers from tuberculosis patients has been evaluated and recommendations are made for handling sputum containers.

Sputum is generally regarded as the clinical specimen most likely to contain large numbers of tubercle bacilli and therefore to present a potential hazard to laboratory staff. The difficulties encountered in the collection of sputum without contaminating the outside of the container, have led to recommendations for disinfecting containers prior to transit to and handling in the laboratory. The United States Public Health Service ${ }^{1}$ recommends wiping the surface of sputum containers with gauze soaked in $5 \%$ phenol. Mitchison $^{2}$ has recommended immersing sputum containers in a suitable disinfectant for $30 \mathrm{~min}$ to ensure that the surface is not infective. The laboratory examination of sputum necessitates the transportation of specimens from hospital clinics or wards to laboratories. Many diagnostic laboratories are attached to hospitals. However, the laboratory diagnosis of tuberculosis is often performed by reference centres, which in developing countries may be located a considerable distance from the clinic. As sputum containers may be handled by numerous personnel during transit it is important to demonstrate that containers may become contaminated with Mycobacterium tuberculosis and ensure that decontamination is reliable. In this investigation sputum specimens collected from tuberculous and nontuberculous patients were examined for evidence of specimen contamination on the outside of the container, and the value and risk of sample contamination from immersing in disinfectant has been evaluated.

Accepted for publication 6 December 1982

\section{Material and methods}

SPUTUM FROM TUBERCULOSIS PATIENTS

Pretreatment sputum from patients admitted to studies of short-course chemotherapy in Hong Kong was collected directly into glass universal containers, packed in sealed containers, and transported to London by air.

\section{SPUTUM SPECIMENS FROM NON-TUBERCULOUS PATIENTS}

Specimens of sputum, collected in plastic universal containers, were received for routine (nontuberculous) bacteriological examination from patients admitted to, or attending, the Hammersmith Hospital.

\section{DETECTION OF TUBERCLE BACILLI ON CONTAINER SURFACES}

During a three-month period (June-August 1981) 317 pretreatment sputum specimens were examined for the presence of Mycobacterium tuberculosis on the outside of the container. Packages containing the sputum containers were opened in a class I microbiological safety cabinet and the containers removed. Each sputum container was inverted at an angle of approximately $45^{\circ}$ over an open bottle containing $10 \mathrm{ml}$ selective Kirchner medium (SK). ${ }^{3}$ The neck of the container, to a depth approximately 10 mm below the cap, was washed with about $6 \mathrm{ml}$ of SK medium using a Pasteur pipette. The washing medium was allowed to drain back into the original medium container, incubated at $37^{\circ} \mathrm{C}$, and examined 
macroscopically at weekly intervals for evidence of growth. Positive cultures were subcultured onto two Löwenstein-Jensen (LJ) slopes which were incubated until sufficient growth was available for further testing. Cultures were identified as $M$ tuberculosis by the production of niacin and failure to grow on medium containing $500 \mathrm{mg} / \mathrm{l}$ 4nitrobenzoate. Negative cultures were discarded after six weeks' incubation. Sputum specimens were also cultured on LJ medium using a modified Petroff decontamination technique. ${ }^{2}$

\section{DETECTION OF SPUTUM CONTAMINATION ON} NON-TUBERCULOUS SPECIMEN CONTAINERS

As few specimens containing $M$ tuberculosis were available, from local patients, sputum specimens from 300 non-tuberculous patients were examined for evidence of sputum contamination of the outside of the container. Specimen containers were swabbed using a cotton wool swab moistened with sterile nutrient broth. Using a rotating motion the neck of the container was swabbed from below the cap to a depth of approximately $10 \mathrm{~mm}$. Swabs were cultured on blood agar medium incubated aerobically at $37^{\circ} \mathrm{C}$ for $48 \mathrm{~h}$. Containers were regarded as contaminated with sputum when the swab culture yielded a moderate or heavy mixed growth of organisms usually found in sputum.

\section{EVALUATION OF DISINFECTION TECHNIQUE}

Glass universal containers were deliberately infected with smear positive tuberculous sputum to simulate maximum contamination of the container. Sterile water $(5 \mathrm{ml})$ was added to each bottle to provide a weight similar to the usual sputum content. A swab stirred in sputum was used to coat the outside of each container from the rim to an area approximately $10 \mathrm{~mm}$ below the shoulder. Two containers were coated with sputum from each of 101 specimens. The caps were replaced and sputum allowed to dry on the containers for $30 \mathrm{~min}$. One container was immersed for $30 \mathrm{~min}$ in a solution of Clearsol (Tenneco Organics Ltd, Bristol) disinfectant diluted 1/45 with cold tap water in accordance with the manufacturer's recommendation. After exposure containers were removed from disinfectant and allowed to dry for $30 \mathrm{~min}$. Each container was then washed with $6 \mathrm{ml} \mathrm{SK}$ medium as previously described except that the total volume of culture medium, into which the washings were collected was increased to $50 \mathrm{ml}$ in order to dilute trace amounts of disinfectant which may have been introduced during washing. To confirm that inhibitory concentrations of disinfectant were not present cultures which were negative after 6 weeks' incubation were inoculated with $0.2 \mathrm{ml}$ of a 10 day Tween albumin culture of a marked strain of $M$ tuberculosis. The culture used was a drug sensitive strain made resistant to rifampicin by heavy inoculation onto $\mathrm{LJ}$ medium containing $64 \mathrm{mg} / \mathrm{l}$ rifampicin. Reinoculated cultures were incubated at $37^{\circ} \mathrm{C}$ for up to 4 wk. Growth was subcultured onto LJ medium containing $64 \mathrm{mg} / \mathrm{l}$ rifampicin in order to detect the marked strain. The second container was washed with SK medium and used as a positive growth control. This growth was identified as $M$ tuberculosis and inoculated onto $\mathrm{LJ}$ medium containing $64 \mathrm{mg} / \mathrm{l}$ rifampicin to ensure that the test strains differed from the marked strain.

DETECTION OF DISINFECTANT INSIDE SPECIMEN CONTAINERS

Although the glass universal container has an adequate seal and does not leak, when tested by the method described in British Standard $5213,{ }^{4}$ it is necessary to ensure that disinfectant does not enter containers during immersion. The test method (BS 5213) was modified in order to detect disinfectant within the containers. Water containing sodium fluorescein at a concentration of $0 \cdot 1 \mathrm{~g} / 1$ was used to dilute Clearsol disinfectant to the recommended strength. In each of three separate tests 35 glass universal containers containing $5 \mathrm{ml}$ water were immersed in dye-labelled Clearsol solution for 30 min. After immersion bottles were rinsed in cold tap water, to remove disinfectant from the outside of the containers, then allowed to drain for $15 \mathrm{~min}$. Containers were then exposed to a source of short wave ultraviolet light to detect penetration of dye-labelled disinfectant.

\section{Results}

TUBERCLE BACILLI ON CONTAINER SURFACES. Using a modified Petroff technique $M$ tuberculosis was isolated from $279(88 \%)$ of the 317 pretreatment sputum specimens examined. Negative or contaminated cultures $(12 \%)$, and corresponding SK cultures, were excluded from the investigation. The SK washing technique resulted in $M$ tuberculosis being isolated from the surface of $18(6.5 \%)$ of 279 culture-positive specimens.

SPUTUM CONTAMINATION OF NON-TUBERCULOUS SPECIMEN CONTAINERS Of the 300 containers examined, using the moist swab technique, $41(14 \%)$ were considered to be contaminated with sputum according to the criteria described. Cultures prepared from uncontaminated containers were usually sterile or yielded a few colonies of micrococci. 
EVALUATION OF DISINFECTION TECHNIQUE

Of 101 control sputum-coated containers washed with SK medium 100 yielded organisms identified as $M$ tuberculosis. All isolates were sensitive to rifampicin $64 \mathrm{mg} / \mathrm{l}$. The remaining culture was contaminated and was excluded from the investigation. Immersion of infected containers in disinfectant was highly successful; none of the 100 containers exposed to Clearsol solution yielded growth in SK medium. There was no evidence to suggest carryover of disinfectant during the washing procedure, all reinoculated cultures yielded growth identified as the marked strain of $M$ tuberculosis demonstrating that they were capable of supporting growth and not therefore significantly contaminated with disinfectant.

\section{PENETRATION OF DISINFECTANT}

Tests were carried out on three occasions. Although traces of fluorescein were detectable on the outside of containers, there was no evidence of dye within the 105 containers tested.

\section{Discussion}

It is clear from the results of this investigation that contamination of specimen container surfaces occurred during the collection of sputum. Viable tubercle bacilli were detected on containers, even after several days in transit, and bacteria indicative of sputum contamination were cultured from containers when specimens were collected locally.

Two widely used manuals give advice on the procedures to be followed when collecting sputum. The United States Department of Health ${ }^{5}$ recommends that patients hold the container to their lower lip and gently release the sample into the container. The International Union against tuberculosis ${ }^{6}$ advises holding the container close to the lips during collection. When this advice is followed the chance of sputum contaminating the container is considerable irrespective of the size of container used. Complex disposable sputum containers have been designed to prevent spillage and contamination, but the cost of such containers prevents their use in many countries.

It has been recommended that all sputum specimens should be processed in a microbiological safety cabinet. ${ }^{7}$ However, this and a previous investigation, ${ }^{8}$ in which tubercle bacilli were cultured from heat-fixed sputum smears, show that laboratory staff may unknowingly handle dried infected sputum without the protection of a safety cabinet. Although droplet nuclei from liquid sputum present the greatest danger to laboratory staff $^{9}$ dried material in which infective units become concentrated may also present a hazard. ${ }^{10}$

In laboratories situated in areas with a low incidence of pulmonary tuberculosis decontamination of sputum containers would seem to be unnecessary, provided sputum specimens are transported to the laboratory in sealed plastic bags and kept separate from the request form. Laboratory techniques used for the examination of sputum from known or suspected tuberculosis patients usually involve considerable handling of the specimen container. It is recommended that a method of containment is used during transportation of high risk specimens and that containers are decontaminated on arrival in the laboratory.

An alternative would be to transfer high risk specimens to a fresh container before commencing laboratory examination, however, this would increase the cost of each investigation and add yet another potentially hazardous manipulation.

\section{References}

${ }^{\prime}$ Laboratory methods for clinical and public health mycobacteriology. United States Department of Health Education and Welfare, 1967.

${ }^{2}$ Mitchison DA. Bacteriology of tuberculosis. Tropical Doctor 1974;4:147-53.

${ }^{3}$ Mitchison DA, Allen BW, Lambert RA. Selective media in the isolation of tubercle bacilli. J Clin Pathol 1973;26:250-2.

${ }^{4}$ BS 5213. Specification for medical specimen containers for microbiology. London: British Standards Institution, 1975.

${ }^{5}$ Laboratory Manual for acid-fast microscopy. US Department of Health, Education and Welfare, 1975.

- Technical guide for sputum examination for tuberculosis by direct microscopy. Bull Int Union Tuberc 1978; suppl 2.

${ }^{7}$ Code of practice for the prevention of infection in clinical laboratories and post-mortem rooms. London: HMSO, 1978.

${ }^{8}$ Allen BW. Survival of tubercle bacilli in heat-fixed sputum smears. J Clin Pathol 1981;34:719-22.

${ }^{9}$ Precautions against tuberculosis infection in the diagnostic laboratory. PHLS Report. Mon Bull Min Hlth and PHLS 1958;17:10-18.

${ }^{10}$ Darlow HM. Safety in the microbiology laboratory. In: Shapton DA, Board RG. Safety in microbiology. London. Academic Press, 1972.

Requests for reprints to: Mr BW Allen, Medical Research Council Unit for Laboratory Studies of Tuberculosis, Royal Postgraduate Medical School, Du Cane Road, London W12 0HS, England. 